\title{
BMJ Open Is there a social gradient of sarcopenia? A meta-analysis and systematic review protocol
}

\author{
Darci Green, ${ }^{1,2}$ Gustavo Duque, ${ }^{1,2}$ Nick Fredman, ${ }^{1}$ Aoun Rizvi, ${ }^{1,2}$ \\ Sharon Lee Brennan-Olsen 1,2,3,4
}

To cite: Green D, Duque G, Fredman N, et al. Is there a social gradient of sarcopenia? A meta-analysis and systematic review protocol. BMJ Open 2018;8:e019088. doi:10.1136/ bmjopen-2017-019088

- Prepublication history and additional material for this paper are available online. To view these files, please visit the journal online (http://dx.doi. org/10.1136/bmjopen-2017019088).

Received 11 August 2017 Revised 25 November 2017 Accepted 12 December 2017

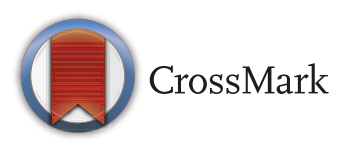

${ }^{1}$ Australian Institute for Musculoskeletal Science (AIMSS), University of Melbourne, Melbourne, Victoria, Australia

${ }^{2}$ Department of MedicineWestern Health, Melbourne Medical School, University of Melbourne, Melbourne, Victoria, Australia

${ }^{3}$ Institute of Health and Aging, Australian Catholic University, Melbourne, Victoria, Australia ${ }^{4}$ Department of Medicine, Deakin University, Geelong, Victoria, Australia

Correspondence to Dr Sharon Lee Brennan-Olsen; sbrennan@unimelb.edu.au

\section{ABSTRACT}

Introduction Sarcopenia (or loss of muscle mass and function) is a relatively new area within the field of musculoskeletal research and medicine. Investigating whether there is a social gradient, including occupation type and income level, of sarcopenia, as observed for other diseases, will contribute significantly to the limited evidence base for this disease. This new information may inform the prevention and management of sarcopenia and widen the evidence base to support existing and future health campaigns.

Methods and analysis We will conduct a systematic search of the databases PubMed, Ovid, CINAHL, Scopus and EMBASE to identify articles that investigate associations between social determinants of health and sarcopenia in adults aged 50 years and older. Eligibility of the selected studies will be determined by two independent reviewers. The methodological quality of eligible studies will be assessed according to predetermined criteria. Established statistical methods to identify and control for heterogeneity will be used, and where appropriate, we will conduct a meta-analysis. In the event that heterogeneity prevents numerical synthesis, a best evidence analysis will be employed. This systematic review protocol adheres to the Preferred Reporting Items for Systematic Reviews and Meta-Analyses Protocols reporting guidelines and will be registered with the International Prospective Register of Systematic Reviews (PROSPER0).

Ethics and dissemination This systematic review will use published data, thus ethical permissions will not be required. In addition to peer-reviewed publication, our results will be presented at (inter)national conferences relevant to the field of sarcopenia, ageing and/ or musculoskeletal health and disseminated both electronically and in print.

PROSPERO registration number CRD42017072253.

\section{INTRODUCTION}

WHO defines the social determinants of health as the conditions in which a person is born, lives, grows, works and ages ${ }^{1}$ : social determinants encompass the distribution of economic and social conditions that influence individual and group differences in health status, and they include factors such as

\section{Strengths and limitations of this study}

- This systematic review will focus on the newly defined disease of sarcopenia; a recent addition to the International Classification of Diseases, Tenth Revision, Clinical Modification.

- The selection of eligible studies and methodological assessment will be independently confirmed by two authors.

- Potential confounders and/or mediators of any observed relationship between social determinants and sarcopenia will be highlighted.

- Potential limitations of this review may be a paucity of data due to the 'newness' of sarcopenia and, plausibly, much heterogeneity in available studies.

area-level or individual-level socioeconomic status, occupation type and level of income. ${ }^{12}$ As such, social determinants have a profound influence in compromising health-related behaviours and health outcomes ${ }^{3}$ : the musculoskeletal system appears to be no exception to this, ${ }^{4-6}$ with the association having already been identified in conditions such as osteoporosis. ${ }^{7}$ There is now a strong evidence base for the role played by social disadvantage on an increased susceptibility to many chronic illnesses, lower uptake or engagement in preventative care and earlier mortality. ${ }^{28}$

Sarcopenia is defined as a progressive and generalised decline in skeletal muscle mass and deficits of overall strength and functionality. ${ }^{9-24}$ Two measures of sarcopenia are currently recommended; a combination of a low level of muscle mass and muscle function (a working definition proposed by the European Working Group on Sarcopenia in Older People $)^{91012}$; or, as an operational definition, muscle mass corrected for height that is two or more SD below that of a young healthy adult is sarcopenia. ${ }^{1325} 26$ Sarcopenia is a relatively new term; although initially coined by Irwin Rosenberg in $1989,{ }^{9}$ it was only added to the International Classification of 
Diseases, Tenth Revision, Clinical Modification in 2016. ${ }^{27}$ Given the current absence of a universal definition of sarcopenia, ${ }^{12} 28$ the nascent literature in this field presents varied estimates of prevalence. ${ }^{17}$ Nonetheless, there is agreement that the main consequences of sarcopenia include frailty, falls, hospitalisation, disability and earlier mortality, ${ }^{9} 10$ thereby increasing the burden on individuals, communities and healthcare systems. ${ }^{920}$

In the context of the 'newness' of sarcopenia and the well-documented role of social determinants in other chronic diseases, we present the protocol for a systematic review, which aims to identify, collate and synthesise the evidence for a social gradient of sarcopenia.

\section{METHODS}

This systematic review protocol has been registered with the International Prospective Register of Systematic Reviews (registration ID: CRD42017072253), and it adheres to the preferred reporting process outlined within the Preferred Reporting Items for Systematic Reviews and Meta-Analyses Protocols (PRISMA-P) 2015 guidelines. $^{29}$

\section{Criteria for considering studies in this review}

Articles that investigate, in adult populations aged 50 years or older, the association between social determinants as risk factors and the outcome of sarcopenia (regardless of definition), and are epidemiological cohort, cross-sectional and/or case-control studies by design, available in full text, and published in any language will be eligible for inclusion in this review.

Grey literature, conference presentations, opinions or commentaries and randomised controlled trials (RCTs) will be excluded. However, where possible, baseline data from RCTs that apply to social determinants of health and sarcopenia prior to the RCT intervention will be included, as it may be possible that these data may be able to offer relevant cross-sectional information.

\section{Search strategy and data extraction}

An electronic search strategy will be performed to investigate medical, health and the social science databases (PubMed, Ovid, CINAHL and EMBASE) to identify studies that match our eligibility criteria, with no limits set for the year of publication. The following Medical Subject Headings will be applied '[All Fields]: (sarcopenia OR aging OR muscular atrophy)'; and the following search strategy will be implemented '( (sarcopenia) OR (aging) OR (muscular atrophy) OR (lean mass) OR (musculoskeletal aging)) AND ((social determinants) OR (ses) OR (income) OR (occupation) OR (socioeconomic))'. Keywords of lean mass and musculoskeletal ageing will also be included. For the complete search strategy, please refer to online supplementary file 1 (PubMed search).

Relevant truncation will be applied to each database. One reviewer will perform the search strategy and identify eligible inclusion literature, and two further reviewers
Table 1 Criteria list for the assessment of methodological quality, modified from Lievense et al ${ }^{3031}$

\begin{tabular}{|c|c|c|}
\hline Item & Criterion & $\mathrm{C} / \mathrm{CC} / \mathrm{CS}$ \\
\hline \multicolumn{3}{|c|}{ Study population } \\
\hline 1 & $\begin{array}{l}\text { Uniform point (selection before disease } \\
\text { was present) }\end{array}$ & $\mathrm{C} / \mathrm{CC} / \mathrm{CS}$ \\
\hline 2 & $\begin{array}{l}\text { Cases and controls drawn from the same } \\
\text { population }\end{array}$ & $\mathrm{CC}$ \\
\hline 3 & Participation rate $>80 \%$ for cases/cohort & $\mathrm{C} / \mathrm{CC} / \mathrm{CS}$ \\
\hline 4 & Participation rate $>80 \%$ for controls & $\mathrm{CC}$ \\
\hline \multicolumn{3}{|c|}{ Assessment of risk factor } \\
\hline 5 & Exposure assessment blinded & $\mathrm{C} / \mathrm{CC} / \mathrm{CS}$ \\
\hline 6 & $\begin{array}{l}\text { Exposure measure identical for cases and } \\
\text { controls }\end{array}$ & $\mathrm{CC}$ \\
\hline 7 & Exposure assessed prior to the outcome & $\mathrm{C} / \mathrm{CC} / \mathrm{CS}$ \\
\hline \multicolumn{3}{|c|}{ Assessment of outcome } \\
\hline 8 & $\begin{array}{l}\text { Outcome assessed identically in studied } \\
\text { populations }\end{array}$ & $\mathrm{C} / \mathrm{CC} / \mathrm{CS}$ \\
\hline 9 & Outcome assessed reproducibly & $\mathrm{C} / \mathrm{CC} / \mathrm{CS}$ \\
\hline 10 & $\begin{array}{l}\text { Outcome assessed according to validated } \\
\text { measures }\end{array}$ & $\mathrm{C} / \mathrm{CC} / \mathrm{CS}$ \\
\hline \multicolumn{3}{|c|}{ Study design } \\
\hline 11 & Prospective design used & $\mathrm{C} / \mathrm{CC}$ \\
\hline 12 & Follow-up time $>12$ months & $\mathrm{C}$ \\
\hline 13 & Withdrawals <20\% & C \\
\hline
\end{tabular}

Analysis and data presentation

$\begin{array}{lll}14 & \text { Appropriate analysis techniques used } & \mathrm{C} / \mathrm{CC} / \mathrm{CS} \\ 15 & \text { Adjusted for at least age and sex } & \mathrm{C} / \mathrm{CC} / \mathrm{CS}\end{array}$

C, applicable to cohort studies; CC, applicable to case-control studies; CS, applicable to cross-sectional studies.

will endorse those articles identified as eligible for inclusion. Professional interpreter assistance will be sought to confirm eligibility for articles written in a language other than English. The eligibility of studies will be checked in a three-step process: (1) assessing titles and abstracts, (2) assessing full-text papers and (3) hand-searching reference lists. The reference lists of the studies that have been deemed eligible will be independently hand-searched by two reviewers. One reviewer will extract data from studies deemed eligible for inclusion, and a further reviewer will cross-check all extracted data. Should disagreement arise regarding those data, a third will provide the final judgement.

\section{Assessment of methodological quality of included articles}

A modified scoring system from Lievense $e t a \vec{l}^{30} 31$ will be employed to analyse data extracted from included studies (table 1). Each of the eligible studies will be scored based on the previously mentioned methodological assessment criteria, and the score given will be either positive or negative for each of the criterion within the study. Currently, no validated criteria exist for assessing 
the methodological quality of observational studies; the Lievense $e t a p^{3031}$ tool provides a reproducible process and enables both validity and informativeness to be assessed in cohort, case-control and cross-sectional study designs, as we have previously demonstrated in the musculoskeletal research field. ${ }^{32-34}$ Those studies deemed eligible will be independently scored by two reviewers using the Lievense $e t a l^{031}$ scoring system: should any differences in scores be found to be irreconcilable, a third reviewer will be introduced and provide the final judgement. The final score will be calculated (as a percentage) and ranked and deemed as having higher methodological quality if scored above the median of the total scores.

\section{Presenting and reporting results}

This systematic review protocol adheres to the PRISMA-P reporting guidelines. ${ }^{35}$ An adapted Quality of Reporting of Meta-analyses standards (QUORUM) diagram ${ }^{36}$ will be used to present study selection, numbers and reasons for inclusion and exclusion of articles in line with the predetermined eligibility criteria. All relevant studies will have key information regarding sarcopenia and social determinants identified, manually extracted and presented, which will be from the articles eligible for inclusion. The key information to be extracted from eligible papers may include, but will not be limited to, the definition and measures of sarcopenia, lean mass and hand grip strength, socioeconomic status, income and education attainment. Results from the methodological scoring will be presented as a percentage.

Where methodological heterogeneity is low, a meta-analysis will be performed and a numerical synthesis presented. The determination of whether statistical heterogeneity is low enough to conduct a meta-analysis will be assessed using the Cochrane $Q$ statistic and the $\mathrm{I}^{2}$ statistic: this method will also inform whether we adopt a fixed-effects or random-effects model. We will apply a threshold of an $\mathrm{I}^{2}>50 \%$ to classify moderate heterogeneity and, if there is overall consistency in the direction of effect, the potential use of a random-effects model. Sources of heterogeneity will be investigated by removing studies at high risk of bias and comparing summary estimates from different study-level methodological characteristics (such as sarcopenia definitions, study design and age of population), using metaregression where appropriate. Funnel plots will be used to investigate publication bias, whereby the distribution of ORs will be presented according to sample size.

Where missing data are identified and to enable methodological scoring using the Lievense et a $\hat{P}^{30} 31$ multiitem instrument, we will (1) employ inverse probability weighting and (2) report the number of missing items on which the inferences were based. ${ }^{37}$ We will present all available data in the eligible studies, noting where missing data preclude our ability to report.

A 'best evidence syntheses' will be undertaken where a meta-analysis is not possible, due to heterogeneity: this latter method will enable an evaluation of the combined
Table 2 Criteria for determining evidence level for best evidence synthesis: modified from Lievense et $a^{30}$

\begin{tabular}{|c|c|}
\hline Level of evidence & $\begin{array}{l}\text { Criteria for inclusion in best } \\
\text { evidence synthesis }\end{array}$ \\
\hline Strong evidence & $\begin{array}{l}\text { Generally consistent findings in: } \\
\text { - Multiple high-quality cohort studies }\end{array}$ \\
\hline Moderate evidence & $\begin{array}{l}\text { Generally consistent findings in: } \\
\text { and high-quality cohort study } \\
\text { and }>2 \text { high-quality case-control } \\
\text { studies } \\
\text { > }>3 \text { high-quality case-control studies }\end{array}$ \\
\hline Limited evidence & $\begin{array}{l}\text { Generally consistent findings in: } \\
\text { A single cohort study } \\
\text { - } 1 \text { or } 2 \text { case-control studies } \\
\text { Multiple cross-sectional studies }\end{array}$ \\
\hline Conflicting evidence & $\begin{array}{l}\text { Inconsistent findings in }<75 \% \text { of the } \\
\text { trials }\end{array}$ \\
\hline No evidence & No studies could be found \\
\hline
\end{tabular}

level of evidence; ranging from no evidence to strong evidence (table 2), as previously published in the musculoskeletal field. ${ }^{33} 34$

\section{Dissemination}

Findings from this review will be published in a peer-reviewed scientific journal and presented at (inter)national conferences relevant to the field of sarcopenia and/or musculoskeletal health.

\section{Ethics}

Given that this systematic review will use only published data, ethical permissions will not be required. However, ethical and governance standards with regard to data management and the presentation and discussion of our findings will be strictly abided by within our research processes.

\section{CONCLUSION}

To the best of our knowledge, this will be the first review to explore whether there is a social gradient of sarcopenia. Investigating sarcopenia in relation to the social determinants of health is a novel area of enquiry, and these findings will contribute to the current paucity of literature regarding the prevention of sarcopenia, while widening the evidence base to support existing and future health campaigns targeted at sarcopenia.

Contributors DG, GD and SLB-0 conceptualised the research question for this protocol. All authors edited and revised the research question. DG and SLB-0 contributed to the development of, and confirmed, the research strategy and developed the methodological process. All authors edited, revised and approved the methodological process. DG and SLB-0 drafted the manuscript. All authors edited and contributed to the writing of this paper, read and approved the final version and guaranteed the review.

Funding SLB- 0 is supported by a National Health and Medical Research Council (of Australia) Career Development Fellowship (1107510). 
Competing interests SLB-0 has received speaker fees from Amgen. GD has received speaker fees from Amgen, Sanofi and Eli Lilly Australia.

Provenance and peer review Not commissioned; externally peer reviewed.

Open Access This is an Open Access article distributed in accordance with the Creative Commons Attribution Non Commercial (CC BY-NC 4.0) license, which permits others to distribute, remix, adapt, build upon this work non-commercially, and license their derivative works on different terms, provided the original work is properly cited and the use is non-commercial. See: http://creativecommons.org/ licenses/by-nc/4.0/

(C) Article author(s) (or their employer(s) unless otherwise stated in the text of the article) 2018. All rights reserved. No commercial use is permitted unless otherwise expressly granted.

\section{REFERENCES}

1. WHO. Social determinants of health: World Health Organization. 2017. http://www.who.int/social_determinants/en/ (accessed 18 Jul 2017).

2. Sullivan SS, Mistretta F, Casucci S, et al. Integrating social context into comprehensive shared care plans: a scoping review. Nurs Outlook 2017;65:597-606.

3. Zhang A, Padilla YC, Kim Y. How early do social determinants of health begin to operate? results from the fragile families and child wellbeing study. J Pediatr Nurs 2017;37:42-50.

4. Brennan SL, Holloway KL, Williams LJ, et al. The social gradient of fractures at any skeletal site in men and women: data from the geelong osteoporosis study fracture grid. Osteoporos Int 2015;26:1351-9.

5. Brennan SL, Yan L, Lix LM, et al. Sex- and age-specific associations between income and incident major osteoporotic fractures in Canadian men and women: a population-based analysis. Osteoporos Int 2015;26:59-65.

6. Brennan SL, Leslie WD, Lix LM. Associations between adverse social position and bone mineral density in women aged 50 years or older: data from the Manitoba Bone Density Program. Osteoporos Int 2013;24:2405-12.

7. Brennan-Olsen SL, Page RS, Berk M, et al. DNA methylation and the social gradient of osteoporotic fracture: a conceptual model. Bone 2016;84:204-12.

8. Chen C, Weider K, Konopka K, et al. Incorporation of socioeconomic status indicators into policies for the meaningful use of electronic health records. J Health Care Poor Underserved 2014;25:1-16.

9. Antunes AC, Araújo DA, Veríssimo MT, et al. Sarcopenia and hospitalisation costs in older adults: a cross-sectional study. Nutr Diet 2017;74:46-50.

10. Bianchi L, Ferrucci L, Cherubini A, et al. The predictive value of the EWGSOP definition of sarcopenia: results from the InCHIANTI Study. $J$ Gerontol A Biol Sci Med Sci 2016;71:259-64.

11. Charest-Morin R, Street J, Zhang H, et al. Frailty and Sarcopenia do not predict adverse events in an elderly population undergoing noncomplex primary elective surgery for degenerative conditions of the lumbar spine. Spine J 2017 https://doi.org/.

12. Cruz-Jentoft AJ, Baeyens JP, Bauer JM, et al. Sarcopenia: European consensus on definition and diagnosis: report of the European working group on Sarcopenia in older people. Age Ageing 2010;39:412-23.

13. Janssen I. Evolution of sarcopenia research. Appl Physiol Nutr Metab 2010;35:707-12.

14. Landi F, Liperoti R, Russo A, et al. Sarcopenia as a risk factor for falls in elderly individuals: results from the iISIRENTE study. Clin Nutr 2012;31:652-8.
15. Lang T, Streeper T, Cawthon P, et al. Sarcopenia: etiology, clinical consequences, intervention, and assessment. Osteoporos Int 2010;21:543-59.

16. Morley JE, Abbatecola AM, Argiles JM, et al. Sarcopenia with limited mobility: an international consensus. J Am Med Dir Assoc 2011;12:403-9.

17. Shaw SC, Dennison EM, Cooper C. Epidemiology of sarcopenia: determinants throughout the lifecourse. Calcif Tissue Int 2017;101:229-47.

18. Stoever K, Heber A, Eichberg S, et al. Sarcopenia and Predictors of Skeletal Muscle Mass in Elderly Men With and Without Obesity. Gerontol Geriatr Med 2017;3:233372141771363.

19. Cruz-Jentoft AJ, Landi F, Schneider SM, et al. Prevalence of and interventions for sarcopenia in ageing adults: a systematic review. Report of the International Sarcopenia Initiative (EWGSOP and IWGS). Age Ageing 2014;43:748-59.

20. Mijnarends DM, Schols JMGA, Halfens RJG, et al. Burden-of-illness of Dutch community-dwelling older adults with sarcopenia: Health related outcomes and costs. Eur Geriatr Med 2016;7:276-84.

21. Joseph C, Kenny AM, Taxel P, et al. Role of endocrine-immune dysregulation in osteoporosis, sarcopenia, frailty and fracture risk. Mol Aspects Med 2005;26:181-201.

22. Phu S, Boersma D, Duque G. Special Section: Sarcopenia: Exercise and Sarcopenia. J Clin Densitom 2015;18:488-92.

23. Baumgartner RN, Waters DL, Gallagher D, et al. Predictors of skeletal muscle mass in elderly men and women. Mech Ageing Dev 1999;107:123-36.

24. Scott D, Seibel M, Cumming R, et al. Sarcopenic obesity and its temporal associations with changes in bone mineral density, incident falls, and fractures in older men: the concord health and ageing in men project. J Bone Miner Res 2017;32:575-83.

25. Baumgartner RN, Koehler KM, Gallagher D, et al. Epidemiology of sarcopenia among the elderly in New Mexico. Am J Epidemiol 1998;147:755-63.

26. Levinger I, Phu S, Duque G. Sarcopenia and osteoporotic fractures. Clin Rev Bone Miner Metab 2016;14:38-44.

27. Anker SD, Morley JE, von Haehling S. Welcome to the ICD-10 code for sarcopenia. J Cachexia Sarcopenia Muscle 2016;7:512-4.

28. Beaudart $C$, Rizzoli R, Bruyère $O$, et al. Sarcopenia: burden and challenges for public health. Arch Public Health 2014;72.

29. Moher D, Shamseer L, Clarke M, et al. Preferred Reporting Items for Systematic review and Meta-Analysis protocols (PRISMA-P) 2015 statement. Syst Rev 2015;4:1.

30. Lievense AM, Bierma-Zeinstra SM, Verhagen AP, et al. Influence of obesity on the development of osteoarthritis of the hip: a systematic review. Rheumatology 2002;41:1155-62.

31. Lievense A, Bierma-Zeinstra S, Verhagen A, et al. Influence of work on the development of osteoarthritis of the hip: a systematic review. $J$ Rheumatol 2001;28:2520-8.

32. Brennan-Olsen SL, Quirk SE, Leslie WD, et al. Comparison of fracture rates between indigenous and non-indigenous populations: a systematic review protocol. BMJ Open 2016;6:e012124-e24.

33. Brennan SL, Pasco JA, Urquhart DM, et al. The association between urban or rural locality and hip fracture in community-based adults: a systematic review. J Epidemiol Community Health 2010;64:656-65.

34. Brennan SL, Pasco JA, Urquhart DM, et al. The association between socioeconomic status and osteoporotic fracture in population-based adults: a systematic review. Osteoporos Int 2009;20:1487-97.

35. Moher D, Shamseer L, Clarke M, et al. Preferred Reporting Items for Systematic review and Meta-Analysis protocols (PRISMA-P) 2015 statement. Syst Rev 2015;4:148-60.

36. Moher D, Cook DJ, Eastwood S, et al. Improving the quality of reports of meta-analyses of randomised controlled trials: the QUOROM statement. The Lancet 1999;354:1896-900.

37. Eekhout I, de Boer RM, Twisk JW, et al. Missing data: a systematic review of how they are reported and handled. Epidemiology 2012;23:729-32. 